\title{
The Left Atrial Septal Pouch: A New Stroke Risk Factor?
}

\author{
Ruchi Kapoor ${ }^{1,2} \cdot$ Lara Wadi $^{3,4}$ - Brian Becerra ${ }^{2}$ - Michael Eskander ${ }^{5}$. Ali Razmara ${ }^{3}$. Dawn Lombardo ${ }^{2}$. \\ Annlia Paganini-Hill ${ }^{3}$. Jin Kyung Kim ${ }^{2} \cdot$ Mark Fisher $^{3,6}$
}

Received: 21 April 2020 / Revised: 31 August 2020 / Accepted: 16 September 2020 / Published online: 4 January 2021

(C) The Author(s) 2021

\begin{abstract}
The left atrial septal pouch (LASP) occurs due to incomplete fusion of septa primum and secundum at the inter-atrial septum, creating an open flap that may serve as a thromboembolic source. Prior studies have demonstrated increased prevalence of LASP in cryptogenic strokes. The aim of the current study was to validate the above findings in a separate, larger group of stroke and non-stroke patients. We examined transesophageal echocardiograms (TEEs) performed between July 2011 and December 2018. LASP prevalence was determined in TEEs referred for ischemic stroke or transient ischemic attack ("stroke") and compared with LASP prevalence in patients undergoing TEEs for other reasons ("non-stroke"). Stroke subtyping was performed using the Trial of Org 10172 in Acute Stroke Treatment (TOAST) criteria. There were 306 TEEs from 144 non-stroke and 162 stroke patients. Mean age and sex distribution were $56 \pm 1$ (mean \pm SE) and 65\% male in the non-stroke group and $58 \pm 1$ and $54 \%$ male in the stroke group. The overall prevalence of LASP was 31\%. The prevalence of LASP was $28 \%$ (41/144) in non-stroke patients, $25 \%$ (24/95) in non-cryptogenic stroke patients, and 43\% (29/67) in cryptogenic stroke patients. LASP prevalence was significantly higher in the cryptogenic subgroup compared with the non-cryptogenic subgroup $(p=0.02)$. These findings demonstrate a significant association of LASP with risk of cryptogenic stroke, suggesting that LASP may serve as a thromboembolic nidus. Additional studies are needed to determine the generalizability of these findings, and their therapeutic implications, supporting LASP as a stroke risk factor.
\end{abstract}

Keywords Cryptogenic stroke $\cdot$ Inter-atrial septum $\cdot$ Ischemic stroke $\cdot$ Left atrial septal pouch $\cdot$ Transesophageal echocardiogram

\section{Introduction}

Cryptogenic strokes account for up to $30-40 \%$ of all ischemic strokes, and a significant subset is thought to originate from

Mark Fisher

mfisher@hs.uci.edu

1 Department of Medicine, Cardiology, University of Washington, Seattle, WA, USA

2 Department of Medicine, Cardiology, University of California Irvine, Orange, CA, USA

3 Department of Neurology, University of California Irvine, Orange and Irvine, CA, USA

4 Department of Medicine, Duke University, Durham, NC, USA

5 Department of Medicine, Cardiology, University of California San Diego, San Diego, CA, USA

6 Department of Neurology, UC Irvine Medical Center, 101 The City Drive South, Building 55, Room 121, Orange, CA 92868, USA distant emboli despite exclusion of arrhythmias and intracardiac thrombus [1]. These infarcts are categorized as embolic strokes of undetermined source (ESUS), and their pathophysiology remains incompletely understood. Recent large, randomized trials comparing the efficacy of anticoagulation with antiplatelet therapy failed to demonstrate superiority of anticoagulation over aspirin in preventing recurrent stroke in patients with ESUS [2-4]. In these studies, presence or absence of structural or functional abnormalities of the left atrium that may promote thromboembolism independent of atrial arrhythmias was not examined. Indeed, left atrial dysfunction has been found to be more prevalent in ESUS than in other stroke subtypes $[5,6]$.

Examination of the left atrial structure may help elucidate a mechanism underlying ESUS or cryptogenic stroke. The anatomic variant known as the left atrial septal pouch (LASP) is of special interest as a possible source of emboli in cryptogenic strokes. LASP was first described in 2010 by Krishnan and Salazar as a unique anatomic entity that occurs with incomplete fusion of the inter-atrial septum [7]. In utero, a separation 
between septum primum and septum secundum allows for flow of oxygenated maternal blood into the fetal systemic circulation while bypassing pulmonary circulation. After birth, the left atrial pressure increases causing the gap between the septum primum and secundum to close and fuse over time. However, in $20-30 \%$ of adults, the two septa fail to fuse, leading to patent foramen ovale (PFO). PFO is particularly prevalent in the younger population and suggests that septal remodeling and fusion is a lifelong process [8]. As proposed by Hołda et al., a septal pouch results from partial fusion of a long PFO channel. This forms a diverticulum with a free edge or flap of one septum opening either into the right, left, or both atria [8]. LASP specifically results from fusion just at the caudal edge of the PFO channel, resulting in an opening towards the left atrium (Fig. 1). Nearly $40 \%$ of adults were observed to have LASP in the original study described by Krishnan and Salazar, although subsequent studies report varying prevalence of $11-42 \%$ [8]. The pouch-like structure of LASP can serve as a potential site of thrombus formation, especially in conditions prone to stasis in the left atrium, such as in atrial cardiopathy, atrial fibrillation, or mitral stenosis.

Since the initial reporting of LASP, a series of case studies have shown thrombus attachment to the LASP, including some instances in patients with strokes [9-11]. However, clearly linking the LASP with cryptogenic stroke has been more challenging. Results from previous retrospective studies have been mixed, with some showing a positive association between LASP and cryptogenic stroke [12-14] and others failing to demonstrate such a relationship [15-17]. Although a recent meta-analysis supports a positive association between LASP and cryptogenic stroke [18], interpreting the data collectively from these studies is limited due to multiple factors, including small sample sizes, heterogeneous patient populations, and widely varying LASP prevalence, which may reflect technical limitations of different imaging modalities used for visualizing the inter-atrial septum. The aim of the current study was to investigate the relationship between presence of
LASP and stroke in a large, well-defined population of patients with stroke and control subjects who underwent transesophageal echocardiography (TEE).

\section{Methods}

This was a cross-sectional retrospective study in which all patients who underwent consecutive TEE studies at the University of California Irvine Medical Center between July 2011 to December 2018 were screened for inclusion criteria. All consecutive TEE studies with the indication "stroke," "CVA" (cerebrovascular accident), or "TIA" (transient ischemic attack) in the order requisition were included for this study in the "stroke group." The "non-stroke group" was a subset of TEE studies from the same time period that did not specify "stroke," "CVA," or "TIA" as an indication. Examples of alternative indications included endocarditis, atrial fibrillation or flutter, or valvular disease. Incomplete TEE studies or TEE studies that did not include imaging of the inter-atrial septum were excluded. Patients with intracranial hemorrhage or control subjects with incidental finding of stroke on brain imaging were excluded from analysis. Patient charts were also reviewed for the presence of known vascular risk factors and comorbidities, including remote history of stroke or TIA, atrial fibrillation/flutter, congestive heart failure, coronary artery disease, diabetes mellitus, hypertension, hyperlipidemia, and smoking status.

The TEE images were evaluated for the presence of LASP, atrial septal defects (ASD), or PFO by a cardiology team composed of cardiology fellows and a senior medical resident, who were blinded to the patient demographics and other clinical information including stroke subtyping (inter-observer agreement 77-97\%). An attending cardiologist and Director of the Echocardiography Laboratory (JKK), also blinded to patient information, confirmed the presence of LASP, and only in those with concordance with the original reviewer
Fig. 1 Examples of LASP (arrows) in our patient population. (a) A patient with an open flap directed towards the left atrium. (b) Absence of microbubbles in the left atrium demonstrates a fused septum in this patient with LASP
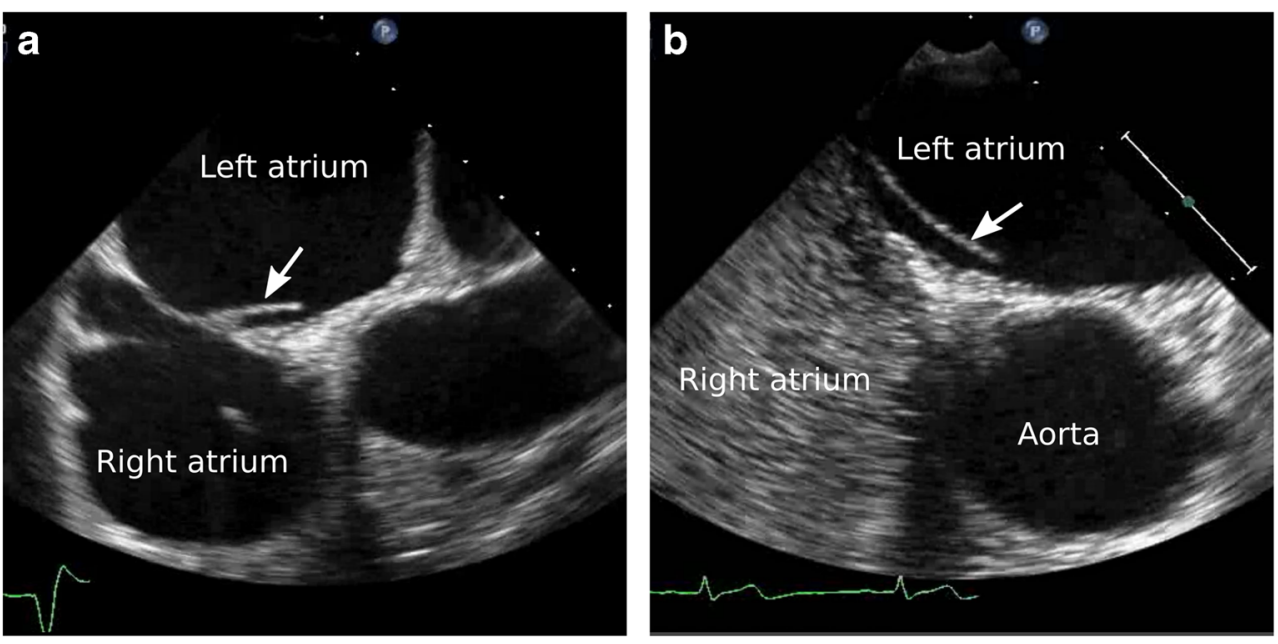
were included in the study. The presence of LASP was evaluated primarily in the bi-caval view (typically obtained with transducer in the mid-esophageal position and angled 90-110 degrees) or in the short-axis view (typically obtained with transducer in the mid-esophageal position and angled 25-45 degrees). LASP was defined as the fusion of septum primum and septum secundum at the caudal end with an open "flap" that created a septal pouch with the opening directed towards the left atrium, and the absence of ASD and PFO. ASD or PFO was excluded by evaluating the morphology of the interatrial septum in 2D views, color Doppler echocardiography, and agitated saline injection with and without Valsalva maneuver to confirm absence of inter-atrial shunt; as in prior studies [12-16], patients with ASD or PFO were excluded from the analysis. The presence of ischemic stroke was verified by review of head and neck imaging (magnetic resonance imaging, magnetic resonance angiography, and/or computed tomography angiography). All ischemic strokes were further subtyped by a vascular neurologist and by a cerebrovascular fellow in accordance with the modified Trial of Org 10172 in Acute Stroke Treatment (TOAST) criteria (inter-observer agreement 96\%, kappa coefficient 0.92) [12, 19].

We compared the prevalence of LASP among non-stroke patients, patients with cryptogenic stroke, and patients with non-cryptogenic stroke. We compared differences in patient characteristics in non-stroke vs. stroke patients, LASP vs. non-LASP patients, and cryptogenic vs. non-cryptogenic stroke patients, using the chi-square test of proportions for categorical variables (Fisher's exact test for $2 \times 2$ tables) and $t$ tests for continuous variables. All tests were two-sided, and a $\mathrm{p}$ value $<0.05$ was considered statistically significant. Univariate and multivariate odds ratio (OR) for stroke and cryptogenic stroke was determined in patients with LASP vs. no LASP. Multivariate analysis was adjusted for age, sex, hypertension, diabetes, hyperlipidemia, atrial fibrillation, coronary artery disease, congestive heart failure, and cardiac thrombus. Analyses were performed using SAS@ version 9.4 (SAS Institute Inc., Cary, NC).

\section{Results}

A total of 306 TEEs from 144 non-stroke and 162 stroke patients were evaluated for this study (Table 1). The nonstroke and stroke groups did not differ significantly by age $(55.9 \pm 1.2$ vs. $58.2 \pm 1.2$ years old, $p=0.18)$ or sex (male $65 \%$ vs. $54 \%, p=0.08$ ). Stroke patients were more likely to have a history of hypertension ( $75 \%$ vs. $58 \%, p=0.002)$. Non-stroke patients were more likely to have a history of atrial fibrillation or flutter ( $33 \%$ vs. $15 \%, p=0.0002)$, congestive heart failure ( $24 \%$ vs. $9 \%, p=0.0003)$, and coronary artery disease $(22 \%$ vs. $12 \%, p=0.045)$. The overall prevalence of LASP in our patient population was $31 \%$ (94 of 306). LASP was present in 41 (28\%) non-stroke patients compared with 53 (33\%) stroke patients $(p=0.46)$. Table 2 shows the patient characteristics by the presence or absence of LASP. There was no statistically significant difference between patients with and without LASP in regard to age, sex, or medical history.

We further evaluated the relationship between LASP and different stroke subtypes based on the modified TOAST criteria (Table 3, Fig. 2). Of the 162 stroke patients in the study, 67 (41\%) had cryptogenic stroke. Of the patients with cryptogenic stroke, $43 \%$ had LASP on their TEEs, which was significantly different from non-stroke patients $(28 \% ; p=$ 0.04). Cardioembolic stroke subgroup followed with the second-highest prevalence of LASP at 37\%. LASP was least associated with large-artery atherosclerosis subtype. Among all non-cryptogenic stroke patients, the prevalence of LASP was $25 \%$, which was significantly lower than $43 \%$ seen in the cryptogenic stroke subgroup ( $p=0.02)$.

Using univariate analysis, we found that the risk of stroke was increased in patients with LASP compared with patients without LASP, but not significantly so (OR 1.21 ; 95\% CI $0.74-1.98$ ). The results were similar when adjusted for common risk factors - age, sex, hypertension, diabetes, hyperlipidemia, atrial fibrillation, coronary artery disease, congestive heart failure, and cardiac thrombus (1.26; 95\% CI 0.73-2.17). Using similar analysis, we found that the risk for cryptogenic stroke was significantly increased in the LASP population (OR 2.15; 95\% CI 1.1-4.21) even after adjusting for common stroke risk factors (OR 2.37; 95\% CI 1.13-4.98).

\section{Discussion}

The current study is a retrospective evaluation of LASP in stroke and non-stroke patients who underwent TEEs and demonstrates a significantly increased risk of cryptogenic stroke in patients with LASP, even after adjusting for common stroke risk factors. The current study confirms and strengthens a prior investigation by our group, which demonstrated that cryptogenic stroke patients are more likely to have LASP compared with non-cryptogenic stroke patients [12]. Our two studies together support this anatomic entity's pathological role in engendering thromboembolic strokes. Our findings are in disagreement with prior investigations that failed to show a correlation between LASP and cryptogenic strokes $[15-17,20]$. However, some of those studies were significantly underpowered compared with the current study, used computerized tomography (CT) scan rather than TEE to evaluate the inter-atrial septum, or exclusively included older patients (>50 years old) who are less likely to have LASP due to ongoing inter-atrial septal remodeling and fusion [8]. The largest study to date evaluated TEEs in 126 cryptogenic stroke and 184 non-stroke patients and demonstrated an overall LASP prevalence of $48 \%$ in their study population [14]. 
Table 1 Characteristics of patients included in this study by stroke status

\begin{tabular}{|c|c|c|c|}
\hline Characteristic & Non-stroke $(n=144)$ & Stroke $(n=162)$ & $p$ value \\
\hline Age- years $($ mean \pm SE) & $55.9 \pm 1.2$ & $58.2 \pm 1.2$ & 0.18 \\
\hline Male sex-no. $(\%)$ & $93(65 \%)$ & $88(54 \%)$ & 0.08 \\
\hline \multicolumn{4}{|l|}{ Medical history-no. (\%) } \\
\hline Hypertension $\dagger$ & $84(58 \%)$ & $121(75 \%)$ & $0.002 *$ \\
\hline Diabetes mellitus & $60(42 \%)$ & $60(37 \%)$ & 0.41 \\
\hline Hyperlipidemia $\dagger$ & $56(39 \%)$ & $77(48 \%)$ & 0.13 \\
\hline Atrial fibrillation/flutter & $47(33 \%)$ & $24(15 \%)$ & $0.0002 *$ \\
\hline Coronary artery disease & $31(22 \%)$ & $20(12 \%)$ & $0.045^{*}$ \\
\hline Congestive heart failure & $35(24 \%)$ & $14(9 \%)$ & $0.0003 *$ \\
\hline Prior stroke & & $35(22 \%)$ & \\
\hline Prior TIA & & $8(5 \%)$ & \\
\hline Cardiac thrombus - no. $(\%)$ & $5(3 \%)$ & $8(5 \%)$ & 0.58 \\
\hline LASP-no. $(\%)$ & $41(28 \%)$ & $53(33 \%)$ & 0.46 \\
\hline
\end{tabular}

${ }^{\dagger}$ One patient with missing history

* Statistically significant difference based on $p<0.05$
Similar to our results, they also show that patients with cryptogenic strokes were more likely to have LASP than nonstroke patients, but they did not compare cryptogenic stroke to other non-cryptogenic stroke subtypes.

It has been suggested that the structure of the LASP is in essence a smaller left atrial appendage which may predispose towards blood stasis and thrombus formation [9, 21]. Gurudevan et al. proposed that right upper pulmonary vein flow is laminar and thus generally protective against thrombus formation in the LASP until another predisposing factor (such as high filling pressures) disrupts the brisk pulmonary vein flow and leads to stasis near the inter-atrial septum. Most case reports demonstrating LASP thrombus are found in patients with another cardiac abnormality, such as valvular disease or left ventricular dysfunction, and thus are in agreement with the above hypothesis $[9,22-26]$. In contrast, there are also isolated cases of stroke patients with LASP thrombus who lack predisposing factors $[10,11]$. This suggests that the presence of LASP by itself may be a predisposing factor in thrombus formation and may suffice as a source of embolism in a subset of cryptogenic stroke patients. Interestingly, Hołda et al. note increased association of LASP with atrial fibrillation [27], whereas in our patient population, atrial fibrillation was more prevalent in patients without LASP $(25 \%$ in patients without LASP vs. $18 \%$ in patients with LASP, see Table 2). The reasons for the different results are unclear as the overall prevalence of LASP $(31 \%$ in our study vs. $36 \%$ in Hołda et al.) and atrial fibrillation (23\% in our study vs. $36 \%$ in Hołda et al.) is similar in both studies. This warrants further investigation into whether LASP contributes to increased stroke risk by engendering atrial cardiomyopathy or arrhythmias.
Table 2 Characteristics of patients included in this study by presence/absence of LASP

\begin{tabular}{llll}
\hline Characteristic & No LASP $(n=212)$ & LASP $(n=94)$ & $p$ value \\
\hline Age-years (mean \pm SE) & $56.9 \pm 1.0$ & $57.6 \pm 1.6$ & 0.72 \\
Male sex-no. $(\%)$ & $122(57 \%)$ & $59(63 \%)$ & 0.45 \\
Medical history—no. $(\%)$ & & & \\
Hypertension $\dagger$ & $143(67 \%)$ & $62(67 \%)$ & 0.90 \\
Diabetes mellitus & $83(39 \%)$ & $37(39 \%)$ & 1.00 \\
Hyperlipidemia $\dagger$ & $92(44 \%)$ & $41(44 \%)$ & 1.00 \\
Atrial fibrillation/flutter & $54(25 \%)$ & $17(18 \%)$ & 0.19 \\
Coronary artery disease & $35(17 \%)$ & $16(17 \%)$ & 1.00 \\
Congestive heart failure & $33(16 \%)$ & $16(17 \%)$ & 0.74 \\
Prior history of stroke & $25(12 \%)$ & $10(11 \%)$ & 0.85 \\
Prior history of TIA & $7(3 \%)$ & $1(1 \%)$ & 0.44 \\
Cardiac thrombus-no. $(\%)$ & $8(4 \%)$ & $5(5 \%)$ & 0.55 \\
\hline
\end{tabular}

${ }^{\dagger}$ One patient with missing history 
Table 3 Prevalence of LASP by stroke subtype

\begin{tabular}{lcc}
\hline Stroke subtype & No LASP & LASP \\
\hline Cryptogenic & $38(57 \%)$ & $29(43 \%)$ \\
Cardioembolic & $24(63 \%)$ & $14(37 \%)$ \\
Small vessel & $11(79 \%)$ & $3(21 \%)$ \\
Large vessel & $11(92 \%)$ & $1(8 \%)$ \\
Other & $14(88 \%)$ & $2(13 \%)$ \\
Multiple mechanisms & $11(73 \%)$ & $4(27 \%)$ \\
Cryptogenic & $38(57 \%)$ & $29(43 \%)^{*}$ \\
Non-cryptogenic & $71(75 \%)$ & $24(25 \%)^{*}$ \\
\hline
\end{tabular}

$* p=0.02$ for difference in LASP prevalence between cryptogenic and non-cryptogenic stroke subgroups

The overall prevalence of LASP in this study (31\%) is lower than the prevalence in autopsy studies (39\% [7]; 47\% [8]) but within the range determined by prior TEE-based studies $(11 \%$ to $48 \%$ [18]) and comparable with studies using CT scan (29\% [20]; 37\% [28]). TEE-based evaluation of the LASP has the largest variation in prevalence estimates, likely related to operator and institutional differences in protocols used for image acquisition. Holda et al. evaluated the inter-atrial septum of 146 patients using TEE with different imaging planes and found good agreement between the bi-caval and short-axis views (kappa 0.68) [29]. They found that the bi-caval view was slightly more sensitive in detecting LASP than the short- axis view, though the difference was statistically insignificant. Elsayed et al. further demonstrated that 3D TEE, providing volumetric data otherwise unobtainable, was more sensitive in detecting LASP than 2D TEE [11]. These technical considerations are particularly important because most TEE-based evaluations of LASP, including this study, are retrospective where the primary intent of the echocardiographer at the time of the imaging may not have been to examine the inter-atrial septum for LASP per se. Not every TEE included both bi-caval and short-axis views, or a 3D interrogation of the inter-atrial septum, and it is likely that LASP may have been missed in some instances. While cardiac CT may have high resolution and utility in defining anatomy, an inter-atrial shunt cannot be excluded solely based on the CT-derived anatomic information. Thus, further research is needed to establish the most optimal noninvasive approach to evaluate LASP.

This study is subject to several limitations. Even though this is one of the larger studies to date that evaluates LASP in stroke patients, the number of patients in each subgroup is small and therefore limits our ability to draw conclusions about LASP prevalence in specific subtypes of non-cryptogenic strokes. Moreover, because this is a retrospective cross-sectional study, we are unable to investigate changes in LASP prevalence over time (i.e., ongoing remodeling of the inter-atrial septum as a person ages) or its direct impact on stroke risk.

In conclusion, the current study confirms results from our group and other studies. In combination with a recent meta-

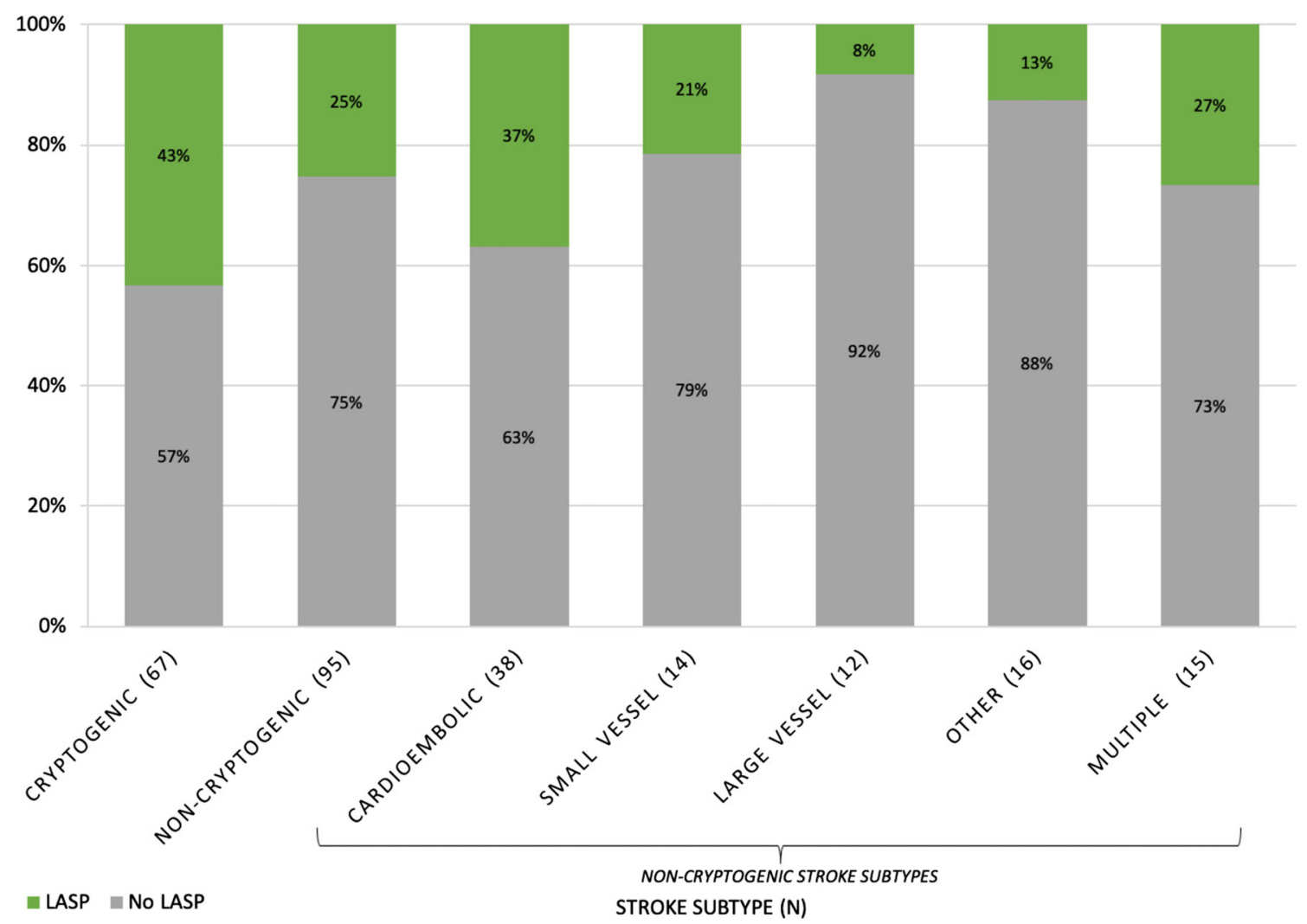

Fig. 2 Prevalence of LASP in different stroke subtypes based on TOAST criteria 
analysis, the data strongly suggest that LASP may be an independent risk factor for cryptogenic strokes [18]. Prospective, longitudinal studies are necessary to better understand the causality of LASP in cryptogenic stroke, further define the mechanism of LASP-related strokes, and investigate targeted treatment options for secondary stroke prevention.

Authors' Contribution The study concept and design were developed by Mark Fisher, Jin Kyung Kim, and Dawn Lombardo. Transesophageal echocardiogram data were collected and reviewed by Michael Eskander, Brian Becerra, Ruchi Kapoor, and Jin Kyung Kim. Electronic medical records were reviewed, and data on patient demographics, stroke history, and imaging were collected by Ali Razmara and Lara Wadi. Statistical analysis was provided by Annlia PaganiniHill. The first draft of the manuscript was written by Ruchi Kapoor, Lara Wadi, and Annlia Paganini-Hill. All authors commented on prior versions of the manuscript and approved the final manuscript.

Data Availability By request

\section{Compliance with Ethical Standards}

Conflicts of Interest The authors declare that they have no conflict of interest.

Ethics Approval This research study was conducted retrospectively from data obtained for clinical purposes and was done in accordance with the ethical standards laid down in the 1964 Declaration of Helsinki and its later amendments. Approval of the study design was obtained from University of California Irvine Institutional Review Board (HS \# 20184733) prior to proceeding with the study.

Consent to Participate A waiver of informed consent was obtained from the Institutional Review Board. All data were collected retrospectively, and patient identifiers were removed prior to analysis.

\section{Consent for Publication Not applicable.}

\section{Code Availability Not applicable.}

Open Access This article is licensed under a Creative Commons Attribution 4.0 International License, which permits use, sharing, adaptation, distribution and reproduction in any medium or format, as long as you give appropriate credit to the original author(s) and the source, provide a link to the Creative Commons licence, and indicate if changes were made. The images or other third party material in this article are included in the article's Creative Commons licence, unless indicated otherwise in a credit line to the material. If material is not included in the article's Creative Commons licence and your intended use is not permitted by statutory regulation or exceeds the permitted use, you will need to obtain permission directly from the copyright holder. To view a copy of this licence, visit http://creativecommons.org/licenses/by/4.0/.

\section{References}

1. Yaghi S, Bernstein RA, Passman R, Okin PM, Furie KL. Cryptogenic stroke: research and practice. Circ Res. 2017;120: $527-40$.
2. Diener H-C, Bernstein R, Hart R. Secondary stroke prevention in cryptogenic stroke and embolic stroke of undetermined source (ESUS). Curr Neurol Neurosci Rep. 2017;17:64.

3. Diener H-C, Sacco RL, Easton JD, Granger CB, Bernstein RA, Uchiyama S, et al. Dabigatran for prevention of stroke after embolic stroke of undetermined source. N Engl J Med. 2019;380:1906-17.

4. Ntaios G, Swaminathan B, Berkowitz SD, Gagliardi RJ, Lang W, Siegler JE, et al. Efficacy and safety of rivaroxaban versus aspirin in embolic stroke of undetermined source and carotid atherosclerosis. Stroke. 2019;50:2477-85.

5. Yaghi S, Kamel H, Elkind MSV. Atrial cardiopathy: a mechanism of cryptogenic stroke. Expert Rev Cardiovasc Ther. 2017;15:5919.

6. Jalini S, Rajalingam R, Nisenbaum R, Javier AD, Woo A, Pikula A. Atrial cardiopathy in patients with embolic strokes of unknown source and other stroke etiologies. Neurology. 2019;92:e288-94.

7. Krishnan SC, Salazar M. Septal pouch in the left atrium: a new anatomical entity with potential for embolic complications. JACC Cardiovasc Interv. 2010;3:98-104.

8. Hołda MK, Koziej M, Hołda J, Piątek K, Tyrak K, Chołopiak W, et al. Atrial septal pouch - morphological features and clinical considerations. Int J Cardiol. 2016;220:337-42.

9. Gurudevan SV, Shah H, Tolstrup K, Siegel R, Krishnan SC. Septal thrombus in the left atrium: is the left atrial septal pouch the culprit? JACC Cardiovase Imaging. 2010;3:1284-6.

10. Wong JM, Lombardo D, Handwerker J, Fisher M. Cryptogenic stroke and the left atrial septal pouch: a case report. J Stroke Cerebrovasc Dis Elsevier Ltd; 2014;23:564-5.

11. Elsayed M, Hsiung MC, Meggo-Quiroz LD, Elguindy M, Uygur B, Tandon R, et al. Incremental value of live/real time threedimensional over two-dimensional transesophageal echocardiography in the assessment of atrial septal pouch. Echocardiography. 2015;32:1858-67.

12. Wong JM, Lombardo DM, Barseghian A, Dhoot J, Hundal HS, Salcedo J, et al. Left atrial septal pouch in cryptogenic stroke. Front Neurol. 2015;6:57.

13. Sun JP, Meng F, Yang XS, Lee AP-W, Chen M, Zhang B, et al. Prevalence of atrial septal pouch and risk of ischemic stroke. Int J Cardiol. 2016;214:37-40.

14. Hołda MK, Krawczyk-Ożóg A, Koziej M, Sorysz D, Hołda J, Dudek D, et al. Left-sided atrial septal pouch is a risk factor for cryptogenic stroke. J Am Soc Echocardiogr. 2018;31:771-6.

15. Tugcu A, Okajima K, Jin Z, Rundek T, Homma S, Sacco RL, et al. Septal pouch in the left atrium and risk of ischemic stroke. JACC Cardiovasc Imaging. 2010;3:1276-83.

16. Wayangankar SA, Patel JH, Patel B, Stavrakis S, Sivaram CA. Clinical and echocardiographic variables associated with LA septal pouch. JACC Cardiovasc Imaging. 2013;6:833-5.

17. Strachinaru M, Catez E, Jousten I, Pavel O, Janssen C, Morissens $\mathrm{M}$, et al. The left atrial septal pouch as a possible risk factor for stroke. Echocardiography. 2016;33:1016-23.

18. Hołda MK, Koziej M. Left-sided atrial septal pouch as a risk factor of cryptogenic stroke: a systematic review and meta-analysis. Cerebrovasc Dis. 2018;46:223-9.

19. Adams HP, Bendixen BH, Kappelle LJ, Biller J, Love BB, Gordon DL, et al. Classification of subtype of acute ischemic stroke. Definitions for use in a multicenter clinical trial. TOAST. Trial of Org 10172 in Acute Stroke Treatment. Stroke. 1993;24:35-41.

20. Yilmaz M, Vural MG, Karcaaltincaba M, Yoldas TK, Yilmaz MS, Kavalci C. Left-sided atrial septal pouch and risk of cryptogenic stroke. Acta Medica Mediterranea. 2016;32:785-9.

21. Chandrashekhar Y, Narula J. LA septal pouch as a source of thromboembolism: innocent until proven guilty? JACC Cardiovasc Imaging. 2010;3:1296-8.

22. Kuwaki H, Takeuchi M, Kaku K, Haruki N, Yoshitani H, Tamura $\mathrm{M}$, et al. Thrombus attached to the left atrial septal pouch assessed 
on 3-dimensional transesophageal echocardiography. Circ J. 2011;75:2280-1.

23. Strachinaru M, Morissens M, Latifyan S, Costescu I. Left atrial septal pouch thrombus assessed on three-dimensional transoesophageal echocardiography. Eur Heart J Cardiovasc Imaging. 2012;13:967-7.

24. Shimamoto K, Kawagoe T, Dai K, Inoue I. Thrombus in the left atrial septal pouch mimicking myxoma. J Clin Ultrasound. Wiley; 2014;42:185-8.

25. Bandyopadhyay S, Mandana K. Left atrial septal pouch: a potential source of systemic thromboembolism: incidental transesophageal echocardiogram findings. Anesth Analg. 2015;121:59-61.

26. Aggarwal S, Kalavakunta J, Gupta V. Left atrial septal pouch thrombus: a common pathology in an uncommon location. Int $\mathrm{J}$ Cardiol. 2016;212:369-70.

27. Hołda MK, Koziej M, Wszołek K, Pawlik W, Krawczyk-Ożóg A, Sorysz D, et al. Left atrial accessory appendages, diverticula, and left-sided septal pouch in multi-slice computed tomography. Association with atrial fibrillation and cerebrovascular accidents. Int J Cardiol. 2017;244:163-8.

28. Hołda MK, Krawczyk-Ożóg A, Koziej M, Pawlik W, Sorysz D, Wszołek K, et al. Cardiac computed tomography compared with two-dimensional transesophageal echocardiography for the detection and assessment of atrial septal pouches. Int J Card Imaging. 2018;34:1305-13.

29. Hołda MK, Krawczyk-Ożóg A, Koziej M, Sorysz D, Hołda J, Dudek D, et al. Mid-esophageal bicaval versus short-axis view of interatrial septum in two-dimensional transesophageal echocardiography for diagnosis and measurement of atrial septal pouches. Echocardiography. 2018;35:827-33.

Publisher's Note Springer Nature remains neutral with regard to jurisdictional claims in published maps and institutional affiliations. 